\title{
On the Local Implementations of Gauge Symmetries in Local Quantum Theory
}

\author{
Francesco Fidaleo \\ Dipartimento di Matematica, Università di Roma "La Sapienza", I-00185 Roma, Italy
}

\begin{abstract}
Under the general assumptions of quantum field theory in terms of local algebras of field operators fulfilling the split property, we prove that any two local covariant implementations of the gauge group (or, in the case of a connected and simply connected Lie gauge group, any two choices of local current algebras) relative to a pair of double cones $\mathcal{O}_{1}, \mathcal{O}_{2}$, are related by a unitary equivalence induced by a unitary in the algebra of observables localized in $\widetilde{\mathcal{O}}_{2}$ which commutes with all fields localized in $\widetilde{\mathcal{O}}_{1}$, where $\widetilde{\mathcal{O}}_{1}$ is any double cone contained in the interior of $\mathcal{O}_{1}$, and $\widetilde{\mathcal{O}}_{2}$ any double cone containing $\mathcal{O}_{2}$ in its interior.
\end{abstract}

\section{Introduction}

Recently the possibility of implementing locally the symmetries of a local quantum theory has been studied $([1-3])$. One of the main motivations was to give a quantum version of classical Noether's theorem.

In $[1,2]$ a sufficient condition, the split property, is given for the local implementability of gauge transformations. Under this hypothesis, all the other symmetries which are present in the theory can be locally implemented as well (e.g. space-time symmetries and supersymmetric transformations; see [3]). The split property can be grounded on general properties of quantum field theory; see $[4,3]$.

A local implementation of the gauge transformations is a representation of the gauge group in a local field algebra, say $F\left(\mathcal{O}_{2}\right)$, which induces the gauge transformations on the field algebra $F\left(\mathcal{O}_{1}\right)$ associated to a "smaller" region $\mathcal{O}_{1}$.

The aim of this paper is to prove the next result. Two local implementations for the regions $\mathcal{O}_{1}, \mathcal{O}_{2}$, will be equivalent by a "well localized" unitary in the observable algebra.

To simplify, we deal with theories with only localizable charges [16]. We suppose in fact that we have given local net of fields on a Hilbert space $\mathscr{H}$ and a gauge group $G$ representated on $\mathscr{H}$. The local fields generate a field algebra $\mathscr{F}$ and the observable algebra $\mathscr{A}$ is derived from $\mathscr{F}$ by the principle of gauge invariance. 
We assume that the field algebra verifies all the general properties $1-7$ of $[5]^{1}$; for terminology and further details we refer to this paper (see also [6]).

According to this assumption one can prove that there is a one-to-one correspondence between the "physical spectrum"2 of $\mathscr{A}$ and the set $\widehat{G}$ of the irreducible representations of $G$. However these sectors can be described via localized morphisms of $\mathscr{A}$. that

For every double cone $\mathcal{O}^{3}$ and $\sigma \in \hat{G}$, there exists a localized morphism ${ }^{4} \rho$ such

$$
\pi_{\sigma} \equiv \pi_{0} \circ \rho
$$

(see footnote 7 of [6]) where in (1.1) $\pi_{\sigma}$ is in the physical spectrum of $\mathscr{A}$ and $\pi_{0}$ is the vacuum representation.

In this paper the following two assumptions play a fundamental role.

(i) The normal commutation relations for the field algebra: there is $\gamma \in G$ with $\gamma^{2}=e$ so that, if we set

$$
F_{ \pm}=\frac{1}{2}\left(F \pm \alpha_{\gamma}(F)\right)
$$

then

$$
F_{+} F_{+}^{\prime}-F_{+}^{\prime} F_{+}=0, \quad F_{+} F_{-}^{\prime}-F_{-}^{\prime} F_{+}=0, \quad F_{-} F_{-}^{\prime}+F_{-}^{\prime} F_{-}=0,
$$

if $F \in \mathscr{F}\left(\mathcal{O}_{1}\right), F^{\prime} \in \mathscr{F}\left(\mathcal{O}_{2}\right), \mathcal{O}_{1} \subset \mathcal{O}_{2}^{\prime}$.

The $F_{+}, F_{-}$in $(1.3)$ are called respectively the Bose and Fermi part of $F$. Necessarily $\gamma$ is in the center of $G$.

(ii) The split property for the field algebra (see assumption (iii) of [2]): for each pair of double cones with $\mathcal{O}_{1} \subset \subset \mathcal{O}_{2}{ }^{5}$ there exists a type $I$ factor $\mathscr{N}$ such that

$$
\mathscr{F}\left(\mathcal{O}_{1}\right) \subset \mathscr{N} \subset \mathscr{F}\left(\mathcal{O}_{2}\right) \text {. }
$$

Actually the triple $\left\{\mathscr{F}\left(\mathcal{O}_{1}\right), \mathscr{F}\left(\mathcal{O}_{2}\right), \Omega\right\}$ is a standard and split inclusion of von Neumann algebras; necessarily $\mathscr{H}$ is separable ([8] Proposition 1.6).

1.1. Definition Let $\mathcal{O}_{1}, \mathcal{O}_{2}$ be two double cones with $\mathcal{O}_{1} \subset \subset \mathcal{O}_{2}$. A strongly continuous representation $g \rightarrow \mathscr{U}(g)$ of $G$ is called local implementation (of the gauge transformations) for the pair $\mathcal{O}_{1}, \mathcal{O}_{2}$ if

$$
\begin{aligned}
& \mathscr{U}(G) \subset \mathscr{F}\left(\mathcal{O}_{2}\right), \\
& \alpha_{g}(F)=\mathscr{U}(g) F \mathscr{U}(g)^{-1} ; \quad F \in \mathscr{F}\left(\mathcal{O}_{1}\right), \quad g \in G .
\end{aligned}
$$

1 The property 7 is assumed in the strengthened form of [5 Eq. (1.23)]. The property 5 of [5] is redundant in the presence of the normal commutation relations for the field algebra

2 The physical spectrum of $\mathscr{A}$ is the set of equivalence classes of irreducible representations appearing in the defining representation of $\mathscr{A}$

3 A double cone $\mathcal{O}$ is a compact region in Minkowski space such that $\mathscr{O}=x-\bar{V}^{+} \cap y+\bar{V}^{+}, x-y \in V^{+}$. We indicate with $\mathscr{O}^{\prime}$ the causal complement of the double cone $\mathcal{O} ; \mathscr{A}\left(\mathcal{O}^{\prime}\right)$ is the $C^{*}$-algebra generated by all $\mathscr{A}\left(\mathcal{O}_{1}\right)$ with $\mathscr{O}_{1} \subset \mathscr{O}^{\prime}$

${ }^{4} \mathrm{~A}$ morphism $\rho$ is said to be localized in the double cone $\mathcal{O}$ if $\rho(A)=A, A \in \mathscr{A}\left(\mathcal{O}^{\prime}\right)$; see [7]

5 We write $\mathcal{O}_{1} \subset \subset \mathcal{O}_{2}$ if $\mathcal{O}_{1} \subset$ interior $\left(\mathcal{O}_{2}\right)$ 
$\mathscr{U}(g)$ is said to be covariant if

$$
\alpha_{h}(\mathscr{U}(g))=\mathscr{U}\left(h g h^{-1}\right) ; \quad g, h \in G .
$$

As already mentioned, the split property assures the existence of covariant local implementations for the pair $\mathcal{O}_{1}, \mathcal{O}_{2}$ with $\mathcal{O}_{1} \subset \subset \mathcal{O}_{2}$.

We want to prove under our assumptions the following uniqueness theorem for local implementations.

Theorem. If $\mathscr{U}(g), \mathscr{V}(g)$ is a pair of local covariant implementations for the double cones $\mathcal{O}_{1}, \mathcal{O}_{2}$, then we can find a unitary operator in $\mathscr{F}\left(\widetilde{\mathcal{O}}_{1}\right)^{\prime} \cap \mathscr{A}\left(\widetilde{\mathcal{O}}_{2}\right)$ which realizes the unitary equivalence between $\mathscr{U}(g)$ and $\mathscr{V}(g)$. Then pair $\widetilde{\mathcal{O}}_{1}, \widetilde{\mathcal{O}}_{2}$ is chosen so that $\widetilde{\mathcal{O}}_{1} \subset \subset \mathcal{O}_{1}$ and $\mathcal{O}_{2} \subset \subset \widetilde{\mathcal{O}}_{2}$.

The theorem is proved in Sect. 3; in Sect. 2 we discuss some mathematical preliminaries.

According to general principles the most natural approach for a local quantum theory is that where the only input are the observable quantities [15]. One can then describe the superselection structure of localizable charges by a semigroup of endomorphisms determined by the local structure of $\mathscr{A}$ (see $[7,12]$ ).

In this case one can "reconstruct" the field algebra and the compact group of internal symmetry ${ }^{6}$. This algebra of fields verifies, by construction all the structural properties recalled above, including the normal commutation relations and, if all charges are localizable in arbitrarily small regions, also the Reeh-Schlieder property with the exception of the split property (ii) that is postulated here. In this case the above theorem is still valid.

\section{Some Mathematical Results}

The contents of this section have been adapted to our aims from $[9,10]$.

2.1. Definition. Let $\mathscr{M}$ be a von Neumann algebra ${ }^{7}$. A norm-closed linear subspace $H \subset \mathscr{M}$ is said to be a Hilbert space in $\mathscr{M}$ if

(a) for each pair $x, y \in H, x^{*} y \in \mathbb{C} I$,

(b) for every non-zero $a \in \mathscr{M}$ there exists an $x \in H$ with $a x \neq 0$.

By the property (a), $H$ is indeed a Hilbert space. It is easy to prove that, if $\mathscr{M}$ is finite, then $H$ is unidimensional and is spanned by a unitary operator. Conversely, if $\mathscr{M}$ is properly infinite, it contains Hilbert spaces of any denumerable cardinality.

If $\left\{u_{j}\right\}_{j \in J}$ is an orthonormal basis for $H$, then $\left\{u_{j} u_{j}^{*}\right\}_{j \in J}$ are mutually orthogonal projections and $\sum_{j \in J} u_{j} u_{j}^{*}=I$.

An endomorphism $\rho_{H}$ of $\mathscr{M}$ is associated uniquely to $H$ by

$$
\rho_{H}(a)=\sum_{j \in J} u_{j} a u_{j}^{*} ; \quad a \in \mathscr{M}
$$

\footnotetext{
6 The abelian case is well described in [13] while, for the general case, these results are announced in the recent paper [14]

7 For the general theory of von Neumann algebras see, for example [11]
} 
The morphism $\rho_{H}$ is characterized via the equation

$$
\rho_{H}(a) x=x a ; \quad x \in H, \quad a \in \mathscr{M} .
$$

Now we speak about von Neumann algebras carrying a continuous action ${ }^{8}$ of a compact group. Then the pair $\{\mathscr{M}, \alpha\}$ will be a von Neumann algebra together with an action of a compact group $G$. We call this pair a covariant system.

Let $H$ be a Hilbert space in $\mathscr{M}$ globally stable under the action $\alpha$, then the mapping $g \in G \rightarrow \alpha_{g}(x) \in H, x \in H$, defines a strongly continuous unitary representation of the group $G$. We indicate with $\mathscr{H}(\alpha)$ the set of such Hilbert spaces.

Obviously, in our case $\{\mathscr{F}(\mathcal{O}), \alpha\}$ (with $\alpha$ the action of the gauge group) is a covariant system and we can find in $\mathscr{H}(\alpha)$ a Hilbert space $H(\rho)$ carrying a representation of class $\rho$ for every $\rho \in \hat{G}$. $H(\rho)$ is given by

$$
H(\rho)=\{\psi \in \mathscr{F}(\mathcal{O}) \mid \rho(A) \psi=\psi A, \quad A \in \mathscr{A}\}
$$

where $\rho$ is a morphism of class $\sigma$, i.e. verifying (1.13), localized in $\mathcal{O}$ (see Sect. 3 of [6]).

2.2. Definition. Let $\{\mathscr{M}, \alpha\}$ be a covariant system. According to [10], we define the monoidal spectrum of $\alpha, \operatorname{Msp}(\alpha)$ in the following way. One element $\sigma \in \hat{G}$ belongs to $\operatorname{Msp}(\alpha)$ if and only if there exists $H \in \mathscr{H}(\alpha)$ carrying a representation of class $\sigma$. If $\operatorname{Msp}(\alpha)=\hat{G}$ we say that $\alpha$ has complete monoidal spectrum.

Hence the gauge action $\alpha$ on $F(\mathcal{O})$ has complete monoidal spectrum for every double cone $\mathcal{O}$.

According to [9], we only treat covariant system $\{\mathscr{M}, \alpha\}$ with $\mathscr{M} \sigma$-finite and $G$ a separable $^{9}$ compact group.

2.3. Definition. We will say that an action $\alpha$ is dominant when

(a) the fixed-point algebra $\mathscr{M}^{\alpha}$ is properly infinite,

(b) there exists a unitary $V \in \mathscr{M} \otimes \mathscr{B}\left(\mathscr{L}^{2}(G)\right)$ such that

$$
\left(\alpha_{g} \otimes e\right)(V)=V(I \otimes \rho(g)), \quad g \in G,
$$

where $g \rightarrow \rho(g)$ denote the right regular representation of $G$ on $\mathscr{L}^{2}(G)$, the $\mathscr{L}^{2}$-space relative to the normalized Haar measure.

2.4. Proposition. Let $\{\mathscr{M}, \alpha\}$ be a covariant system. Suppose that $\mathscr{M}^{\alpha}$ is properly infinite and $\operatorname{Msp}(\alpha)=\hat{G}$. Then $\alpha$ is dominant.

Proof. We construct a Hilbert space $H \in \mathscr{H}(\alpha)$ carrying a representation unitarily equivalent to the right regular representation. Let $J_{\sigma}$ be an index set for every $\sigma \in \hat{G}$ such that $\left|J_{\sigma}\right|=\operatorname{dim} \sigma$ and $J_{\sigma} \cap J_{\tau}=\varnothing$ if $\sigma \neq \tau$. Let $J=\bigcup_{\alpha \in G} J_{\sigma}$. We choose, for every $j \in J$, a Hilbert space $H_{j} \in \mathscr{H}(\alpha)$ carrying a representation of class $\sigma$ if $j \in J_{\sigma}$. Since $\mathscr{M}^{\alpha}$ is properly infinite, there exists a sequence of isometries $\left\{w_{j}\right\}_{j \in J} \subset \mathscr{M}^{\alpha}$ such that

\footnotetext{
8 A continuous action of a locally compact group $G$ on a von Neumann algebra $\mathscr{M}$ is a homomorphism $\alpha$ of $G$ into $\operatorname{Aut}(\mathscr{M})$ such that the functions $g \in G \rightarrow \alpha_{g}(x) \in \mathscr{M}$ are all $\sigma$-weakly continuous

9 In this paper with separable group we mean a topological group with a countable basis of open neighbourhoods
} 
$\left\{w_{j} w_{j}^{*}\right\}_{j \in J}$ are orthogonal projections with $\sum_{j \in J} w_{j} w_{j}^{*}=I$. Then, by construction, the norm-closed linear span of $\left\{w_{j} H_{j}\right\}_{j \in J}$ is a Hilbert space in $\mathscr{H}(\alpha)$ carrying a representation equivalent to the right regular representation of $G$. The proof now follows from Proposition 3.6 of [10].

Given an action $\alpha$ on $\mathscr{M}$, we can easily construct other actions on $\mathscr{M}$ by perturbing $\alpha$ via unitary cocycles. We need the following

2.5. Definition. Let $\{\mathscr{M}, \alpha\}$ be a covariant system. A strongly continuous function $g \rightarrow a(g)$ of $G$ in the unitary group of $\mathscr{M}$ is called an $\alpha$-cocycle if

$$
a\left(g g^{\prime}\right)=a(g) \alpha_{g}\left(a\left(g^{\prime}\right)\right), \quad g g^{\prime} \in G .
$$

Two $\alpha$-cocycles $a, b$ are said to be equivalent if there exists a unitary $u \in \mathscr{M}$ such that

$$
a(g)=u b(g) \alpha_{g}\left(u^{*}\right), \quad g \in G .
$$

In this case we write $a \cong b$.

If $a$ is an $\alpha$-cocycle, then we can define a new action of $G$ on $\mathscr{M}$ by the formula

$$
(a \alpha)_{g}(A)=a(g) \alpha_{g}(A) a(g)^{*} ; \quad A \in \mathscr{M}, \quad g \in G .
$$

A cocycle is dominant if the corresponding action is dominant. It is easy to prove (see III. 2.4 of [9]) the next result.

2.6. Proposition. Let $a, b$ be two $\alpha$-cocycles. If $a, b$ are dominant then $a \cong b$.

\section{A Uniqueness Theorem for Local Implementations of Gauge Transformations}

In this section we prove the previously announced uniqueness theorem for local implementations. We need some considerations.

We have seen that, for any given double cone $\mathcal{O}$, there is a Hilbert space $H(\rho) \subset$ $\mathscr{F}(\mathcal{O})$ carrying a representation of class $\sigma, \sigma \in G$. Of course $\rho$ is a morphism of class $\sigma$ localized in $\mathcal{O}$. One can prove that, if $\sigma$ is a bosonic (fermionic) sector (i.e. the statistics parameter associated to $\sigma$ is positive (negative); see [6]), then $H(\rho) \subset$ $\mathscr{F}_{+}(\mathcal{O})\left(H(\rho) \subset \mathscr{F}_{-}(\mathcal{O})\right)$, where $\mathscr{F}_{+}(\mathcal{O})$ are respectively the Bose and Fermi part of $\mathscr{F}(\mathcal{O})$. Of course

$$
\mathscr{F}_{ \pm}(\mathcal{O})=\frac{1}{2}\left(e \pm \alpha_{\gamma}\right) \mathscr{F}(\mathcal{O})
$$

and $\gamma \in G$ verifies (i) of the introduction.

Now we consider a pair of double cones with $\mathcal{O}_{1} \subset \subset \mathcal{O}_{2}$. By Theorem 2.1 of [2] there exists $U_{\gamma} \in \mathscr{A}\left(\mathcal{O}_{2}\right)$ which implements $\gamma$ on $\mathscr{F}\left(\mathcal{O}_{1}\right)$. With $\mathcal{O}_{0} \subset \mathcal{O}_{1}^{\prime} \cap \mathcal{O}_{2}$, we define a "locally twisted algebra" $\mathscr{F}^{1 . t .}\left(\mathcal{O}_{0}\right)$ by

$$
\mathscr{F}^{\text {l.t. }}\left(\mathcal{O}_{0}\right)=\mathscr{F}_{+}\left(\mathcal{O}_{0}\right)+U_{\gamma} \mathscr{F}_{-}\left(\mathcal{O}_{0}\right) \text {. }
$$

Hence $\mathscr{F}^{\text {l.t. }}\left(\mathcal{O}_{0}\right) \subset \mathscr{F}\left(\mathcal{O}_{1}\right)^{\prime} \cap \mathscr{F}\left(\mathcal{O}_{2}\right)$. By the previous considerations $\mathscr{F}^{\text {l.t. }}\left(\mathcal{O}_{0}\right)$, and therefore $\mathscr{F}\left(\mathcal{O}_{1}\right)^{\prime} \cap \mathscr{F}\left(\mathcal{O}_{2}\right)$, contains a Hilbert space carrying a representation of class $\sigma, \sigma \in \hat{G}$. Further $\mathscr{A}\left(\mathcal{O}_{0}\right) \subset\left(\mathscr{F}\left(\mathcal{O}_{1}\right)^{\prime} \cap \mathscr{F}\left(\mathcal{O}_{2}\right)\right)^{\alpha}$ and $\mathscr{A}\left(\mathcal{O}_{0}\right)$ is properly infinite except in 
the trivial case (see Remark 2.5 of [1]). Therefore we have proved the following. 3.1. Proposition. Let $\mathcal{O}_{1}, \mathcal{O}_{2}$ be double cones with $\mathcal{O}_{1} \subset \subset \mathcal{O}_{2}$. Then on $\mathscr{F}\left(\mathcal{O}_{1}\right)^{\prime} \cap \mathscr{F}\left(\mathcal{O}_{2}\right)$, the gauge action $\alpha$ is dominant.

We fix our attention on a fixed pair of double cones $\mathcal{O}_{1}, \mathcal{O}_{2}$ with $\mathcal{O}_{1} \subset \subset \mathcal{O}_{2}$. As we have seen, by the split property, the set of local covariant implementations is nonvoid. Choose one of these representations, say $\mathscr{U}(g)$, as reference representation. $\mathscr{U}(g)$, induces, in a natural way, an action $\theta$ of $G$ on $\mathscr{F}\left(\mathcal{O}_{1}\right)^{\prime} \cap \mathscr{F}\left(\mathcal{O}_{2}\right)$ :

$$
\theta_{g}(F)=\mathscr{U}(g) F \mathscr{U}(g)^{-1}, \quad g \in G .
$$

It is immediate that every local implementation $\mathscr{V}(g)$ is related to $\mathscr{U}(g)$ by the formula

$$
\mathscr{V}(g)=X(g) \mathscr{U}(h), \quad g \in G,
$$

where $X(g)$ is a $\theta$-cocycle in $\mathscr{F}\left(\mathcal{O}_{1}\right)^{\prime} \cap \mathscr{F}\left(\mathcal{O}_{2}\right)$.

Further, if $\mathscr{V}(g)$ is covariant, the associated cocycle verifies the analogous covariant property:

$$
\alpha_{h}(X(g))=X\left(h g h^{-1}\right), \quad g, h \in G .
$$

In other words, there is a one-to-one correspondence between $\theta$-cocycles (covariant $\theta$-cocycles) in $\mathscr{F}\left(\mathcal{O}_{1}\right)^{\prime} \cap \mathscr{F}\left(\mathcal{O}_{2}\right)$ and local implementations (local covariant implementations) relative to the pair $\mathcal{O}_{1}, \mathcal{O}_{2}$.

We prove the uniqueness theorem in several steps. We start with the following.

3.2. Proposition. Let $g \rightarrow \mathscr{V}_{i}(g), i=1,2$ be a pair of local implementations (not necessarily covariant) for $\mathcal{O}_{1}, \mathcal{O}_{2}$. Then, for any double cone $\mathcal{O}$ such that $\mathcal{O} \subset \subset \mathcal{O}_{1}$, there exists an unitary $V \in \mathscr{F}(\mathcal{O})^{\prime} \cap \mathscr{F}\left(\mathcal{O}_{2}\right)$ that realizes the equivalence between $\mathscr{V}_{1}, \mathscr{V}_{2}$ :

$$
\mathscr{V}_{2}(g)=V \mathscr{V}_{1}(g) V^{-1}, \quad g \in G
$$

Proof. The actions obtained by perturbing $\theta$ with the cocycles related to the $\mathscr{V}_{i}(g)$ coincide with the actions $\theta_{i}$ naturally induced by $\mathscr{V}_{i}(g)$ on $\mathscr{F}(\mathcal{O})^{\prime} \cap \mathscr{F}\left(\mathcal{O}_{2}\right)$. Furthermore $\theta_{i} \mid \mathscr{F}(\mathcal{O})^{\prime} \cap \mathscr{F}\left(\mathcal{O}_{1}\right)=\alpha$. Hence, by Proposition 3.1, $\operatorname{Msp}\left(\theta_{i}\right)=\hat{G}, i=1,2$. The proof now follows by Proposition 2.4 and $2.6^{10}$.

Note that the last result is independent of the covariance property which will play a fundamental role in what follows.

3.3. Lemma. Let $g \rightarrow \mathscr{V}(g)$ be a local covariant implementation relative to the pair $\mathcal{O}_{1}, \mathcal{O}_{2}$. Suppose that $\mathscr{V}(g)$ is equivalent to the reference representation $\mathscr{U}(g)$ by a unitary $V \in \mathscr{F}\left(\mathcal{O}_{1}\right)^{\prime} \cap \mathscr{F}\left(\mathcal{O}_{2}\right)$. Then $w(g)=V^{-1} \alpha_{g}(V)$ is an $\alpha$-cocycle in $\left(\mathscr{F}\left(\mathcal{O}_{1}\right)^{\prime} \cap\left(\mathscr{F}\left(\mathcal{O}_{2}\right)\right)^{\theta}\right.$.

Proof. It is easy to check that $\alpha$ induces automorphisms on $\left(\mathscr{F}\left(\mathcal{O}_{1}\right)^{\prime} \cap \mathscr{F}\left(\mathcal{O}_{2}\right)\right)^{\theta}$ and $w(g)$ verifies the cocycle property $(2.5)$. But, by covariance $V \mathscr{U}\left(h g h^{-1}\right) V^{-1}=$ $\alpha_{h}(V) \mathscr{U}\left(h g h^{-1}\right) \alpha_{h}(V)^{-1}$, i.e. $\mathscr{U}\left(h g h^{-1}\right) V^{-1} \alpha_{h}(V) \mathscr{U}\left(h g^{-1} h^{-1}\right)=V^{-1} \alpha_{h}(V)$; since for every given $h$, varying $g, h g h^{-1}$ spans $G$, the proof now follows.

10 Of course $A\left(\mathcal{O}_{0}\right) \subset\left(F(\mathcal{O})^{\prime} \cap F\left(\mathcal{O}_{2}\right)\right)^{\theta}, i=1,2$ if $\mathcal{O}_{0} \subset \mathcal{O}^{\prime} \cap \mathcal{O}_{1}$ and $A\left(\mathcal{O}_{0}\right)$ is properly infinite 
3.4. Lemma. Let $w(g)$ be as above. Then there exists a unitary $\widetilde{V} \in\left(\mathscr{F}\left(\mathcal{O}_{1}\right)^{\prime} \cap \mathscr{F}(\mathcal{O})\right)^{\theta}$ such that

$$
w(g)=\widetilde{V} \alpha_{g}\left(\widetilde{V}^{-1}\right) ; \quad g \in G
$$

for every double cone $\mathcal{O}$ with $\mathcal{O}_{2} \subset \subset \mathcal{O}$.

Proof. Set $\mathscr{B}=\left(\mathscr{F}\left(\mathcal{O}_{1}\right)^{\prime} \cap \mathscr{F}(\mathcal{O})\right)^{\theta}$. Then $\mathscr{B} \supset \mathscr{F}\left(\mathcal{O}_{2}\right)^{\prime} \cap \mathscr{F}(\mathcal{O})$ and the action $(w \alpha)$, obtained perturbing $\alpha$ by the cocycle $w(g)$, is equal to $\alpha$ on $\mathscr{F}\left(\mathcal{O}_{2}\right)^{\prime} \cap \mathscr{F}(\mathcal{O})$. The proof now follows as in the Proposition $3.2\left(\mathscr{A}\left(\mathcal{O}_{0}\right) \subset \mathscr{B}^{(w \alpha)}\right.$ if $\left.\mathcal{O}_{0} \subset \mathcal{O}_{2}^{\prime} \cap \mathcal{O}\right)$.

Now we can prove the following

3.5. Proposition. Under the hypothesis in Lemma 3.3 there exists an unitary $U \in \mathscr{F}\left(\mathcal{O}_{1}\right)^{\prime} \cap \mathscr{A}(\mathcal{O})$ such that

$$
\mathscr{V}(g)=U \mathscr{U}(g) U^{-1} ; \quad g \in G .
$$

The double cone $\mathcal{O}$ is chosen so that $\mathrm{O}_{2} \subset \subset \mathcal{O}$.

Proof. Let $\widetilde{V}$ be as in (3.6). Set $U=V \widetilde{V}$. Then it is immediate that $U \in \mathscr{F}\left(\mathcal{O}_{1}\right)^{\prime} \cap \mathscr{A}(\mathcal{O})$ and, for every $g \in G, \mathscr{V}(g)=U \mathscr{U}(g) U^{-1}$.

Combining the results of Proposition 3.2 and 3.5 we immediately obtain the next uniqueness theorem for local covariant implementations.

3.6. Theorem. Let $\mathcal{O}_{1}, \mathcal{O}_{2}$ be a pair of double cones with $\mathcal{O}_{1} \subset \subset \mathcal{O}_{2}$. Let $g \rightarrow \mathscr{V}(g)$, $i=1,2$ two strongly continuous representations of $G$ in $\mathscr{F}\left(\mathcal{O}_{2}\right)$ that verify $(1.15)$ and (1.16). Then, for any pair of double cones $\widetilde{\mathcal{O}}_{1}, \widetilde{\mathcal{O}}_{2}$ with $\widetilde{\mathcal{O}}_{1} \subset \subset \mathcal{O}_{1}$ and $\mathcal{O}_{2} \subset \subset \widetilde{\mathcal{O}}_{2}$, there exists a unitary $U \in \mathscr{F}\left(\widetilde{\mathcal{O}}_{1}\right)^{\prime} \cap \mathscr{A}\left(\widetilde{\mathcal{O}}_{2}\right)$ that realizes the equivalence between $\mathscr{V}_{1}(g)$ and $\mathscr{V}_{2}(g)$ :

$$
\mathscr{V}_{2}(g)=U \mathscr{V}_{1}(g) U^{-1}, \quad g \in G .
$$

A physically relevant case is when $G$ is a Lie group. In this case, differentiating a local covariant implementation relative to the pair $\mathcal{O}_{1}, \mathcal{O}_{2}$, we construct a "local current algebra" as in [2]. However, if $G$ is connected and simply connected, there is a one-to-one correspondence between "local current algebras" for the pair $\mathcal{O}_{1}, \mathcal{O}_{2}$ as in (2.6) of [2] and local covariant implementations for the same pair $\mathcal{O}_{1}, \mathcal{O}_{2}$. Hence we have an analogous uniqueness theorem for the choices of local current algebras.

3.7. Corollary. Let $G$ be a connected and simply connected compact Lie group. Let $u \in \mathscr{L} \rightarrow \mathscr{J}_{u}^{(i)}, i=1,2$ be representations of the Lie algebra $\mathscr{L}$ of $G$ as in (2.6) of [2]. Then there exists unitary $U \in \mathscr{F}\left(\widetilde{\mathcal{O}}_{1}\right)^{\prime} \cap \mathscr{A}\left(\widetilde{\mathcal{O}}_{2}\right)$ such that

$$
\mathscr{J}_{u}^{(2)}=U \mathscr{J}_{u}^{(1)} U^{-1}, \quad u \in \mathscr{L} .
$$

The pair $\tilde{\mathcal{O}}_{1}, \widetilde{\mathcal{O}}_{2}$ is chosen as in Theorem 3.6.

3.8. Concluding Remark. The last result would have a role in the construction, starting with the local current algebras, of local current densities that verify the usual "current algebra hypothesis" in elementary particle physics. See [2] for further details about this problem. 


\section{References}

1. Doplicher, S.: Local aspects of superselection rules. Commun. Math. Phys. 85, 73 (1982)

2. Doplicher, S., Longo, R.: Local aspects of superselection rules, II. Commun. Math. Phys. 88, 399 (1983)

3. Buchholz, D., Doplicher, S., Longo, R.: On Noether's theorem in quantum field theory. Annals of Phys. (to appear)

4. Buchholz, D., Wichmann, E.: Causal independence and energy level density of localized states in quantum field theory. Commun. Math. Phys. 106, 321-344 (1986)

5. Doplicher, S., Haag, R., Roberts, J.: Observables and gauge transformations. Commun. Math. Phys. 13, 1 (1969)

6. Doplicher, S., Roberts, J.: Fields, statistics and non-abelian gauge groups. Commun. Math. Phys. 28, 331 (1972)

7. Doplicher, S., Haag, R., Roberts, J.: Local observables and particle statistics. Commun. Math. Phys. 23, 199 (1971)

8. Doplicher, S., Longo, R.: Invent. Math. 75, 493 (1984)

9. Connes, A., Takesaki, M.: Tohoku Math. J. 29, 473 (1977)

10. Roberts, J.: Symp. Math. 20, 335 (1976)

11. Takesaki, M.: Theory of operator algebras I. Berlin, Heidelberg, New York: Springer 1979

12. Doplicher, S., Haag, R., Roberts, J.: Local observables and particle statistics II. Commun. Math. Phys. 35, 49 (1974)

13. Doplicher, S., Haag, R., Roberts, J.: Fields, observables and gauge transformations. II. Commun. Math. Phys. 15, 173 (1969)

14. Doplicher, S., Roberts, J.: Bull. Am. Math. Soc. 11, 333 (1984)

15. Haag, R., Kastler, D.: An algebraic approach to quantum field theory. J. Math. Phys. 5, 848 (1964)

16. Buchholz, D., Fredenhagen, K.: Locality and the structure of particle states. Commun. Math. Phys. 84, 1 (1982)

Communicated by R. Haag

Received March 29, 1986 